\title{
DICTIONARY WRITING SYSTEMS Y OTRAS HERRAMIENTAS INFORMÁTICAS PARA LA ELABORACIÓN, ADMINISTRACIÓN Y PUBLICACIÓN DE DICCIONARIOS
}

\author{
Ruth Yanira Rubio López \\ Instituto Caro y Cuervo (Colombia) \\ ruth.rubio@caroycuervo.gov.co \\ Jonathan Estiven Bonilla Huérfano \\ Instituto Caro y Cuervo (Colombia) / Universidad de Gante (Bélgica) \\ johnatan.bonilla@caroycuervo.gov.co \\ Julio Alexander Bernal Chávez \\ Instituto Caro y Cuervo (Colombia) \\ julio.bernal@caroycuervo.gov.co
}

Recibido: 26/10/2020 - Aprobado: 29/12/2020 - Publicado: 30/07/2021

DOI: doi.org/10.17533/udea.lyl.n80a20

Resumen: Este texto se centra en describir el funcionamiento, los componentes, las características principales y algunos ejemplos de los Dictionary Writing Systems (DWS), los cuales son herramientas informáticas fundamentales para la escritura y administración de diccionarios. Esto implica la automatización en algunos procesos de escritura de las entradas, el uso de bases de datos de diccionarios para otros propósitos, la facilidad de trabajo en equipo de forma remota, entre otros. Estas funcionalidades se vinculan con el tipo de DWS u otras herramientas, y los tipos de diccionarios o fines para los que fueron diseñados.

Palabras clave: Dictionary Writing Systems; diccionarios; Corpus Query Systems; software.

\section{DICTIONARY WRITING SYSTEMS AND OTHER INFORMATIC TOOLS FOR CREATING, MANAGING, AND PUBLISHING DICTIONARIES}

\begin{abstract}
This work describes the functioning, components, main features, and some examples of Dictionary Writing Systems (DWS), which are essential informatic tools for dictionary writing and management. This implies the automation of some processes of write entries, the use of dictionary databases for other purposes, the ease of teamwork remote working, etc. These functionalities are related to the typology of DWS or other programs and the types of dictionaries or purposes they were designed to address.

Key words: Dictionary Writing Systems; dictionaries; Corpus Query Systems; software.




\section{Introducción}

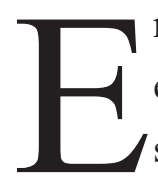

n los años 60 y 70 se inició la aplicación del uso de computadores para la lexicografía; sin embargo, en este tiempo los lexicógrafos no tenían contacto directo con los computadores, ya que la labor lexicográfica

se llevaba a cabo en papel y solo los expertos se encargaban del manejo de los datos (Atkins \& Rundell, 2008). Uno de los primeros lexicógrafos en aplicar la tecnología para la creación de diccionarios fue Laurence Urdang, del Random House Dicionary, que diseñó una base de datos en la que se organizaban los elementos del diccionario por separado para que el computador pudiera hacer la clasificación, organización y verificación de los datos disponibles (Logan, 1991). Posteriormente, uno de los primeros diccionarios en implementar el uso de computadores de forma más avanzada fue la primera edición del Logman Dictionary of Contemporary English (LDOCE) de Paul Procter en 1978. El LDOCE «incluyó algunas categorías de datos (especialmente, un sistema complejo de codificación semántica) que no tenía la intención de aparecer en el diccionario»» ${ }^{1}($ Rundell \& Kilgarriff,, 2011, p. 258). Asimismo, el LDOCE fue uno de los primeros diccionarios en usar un conjunto limitado de palabras de alta frecuencia para escribir las definiciones (Atkins \& Rundell, 2008, p. 112).

A comienzos de los años 80, John Sinclair fundó el Collins Birnmingham University International Language Database (COBUILD), que consistía en la creación de un corpus electrónico de inglés contemporáneo cuyo principal producto de esta investigación sería el COBUILD English Dictionary (Sinclair, 1991). Este fue el primer diccionario basado en datos informáticos recogidos con fines lexicográficos. Más adelante, a principios de los años 90, los lexicógrafos comenzaron a usar computadores directamente para la elaboración de diccionarios y, a mediados esta década, la disminución de los costos de hardware, el aumento de la capacidad de almacenamiento y la llegada del correo electrónico hicieron posible que grupos editoriales separados geográficamente pudieran trabajar en un único diccionario (Atkins \& Rundell, 2008).

Actualmente, los diccionarios se elaboran en computadores con la ayuda de herramientas de software cada vez más específicas que se están desarrollando para este propósito (Abel, 2012). Entre estas se encuentran los Dictionary Writing Systems (DWS), que en español se podrían traducir como «Sistemas Gestores de Diccionarios» (SGS). Los primeros DWS se desarrollaron con el objetivo de facilitar al lexicógrafo la escritura de la entrada (Rundell \& Kilgariff, 2011). Sin embargo, con el tiempo se han desarrollado sistemas cada vez más completos y que cumplen mayores tareas para la labor lexicográfica. Un DWS puede servir para la escritura de la entrada, la asignación de tareas al equipo de lexicógrafos, la definición de los estilos y colores de publicación, entre otras tareas que se revisarán a lo largo de este documento.

Otra de las herramientas más reconocidas para facilitar la labor lexicográfica son los Corpus Query Systems (CQS), que en español se podrían traducir como «Sistemas de Consulta de Corpus» (SCC), y que también son

1. «[...] included some categories of data (notably a complex system of semantic coding) which were never intended to appear in the dictionary itself. » (Rundell \& Kilgarriff, 2011, p. 258). (Traducción propia). 
DWS y otras herramientas informáticas para la elaboración, administración y publicación de diccionarios conocidos por nombres como corpus manager o corpus browser. Los CQS permiten analizar los datos de los corpus de distintas maneras (Atkins \& Rundell, 2008) y pueden estar integrados o no en los DWS. Entre estos se puede mencionar a TshwaneLex (también conocido como TLEX), uno de los sistemas que incluye un CQS en su plataforma.

\section{Los DWS: sus componentes y funcionalidades}

Kilgarriff (2006), en las actas del Fourth International Workshop on Dictionary Writing Systems, definió los DWS como software para la escritura y producción de diccionarios. Por su parte, Atkins y Rundell (2008) lo definen como un programa que permite a los lexicógrafos compilar y editar diccionarios, facilita la administración de estos y la publicación en formato impreso o electrónico. Grundy y Rawlinson (2016) señalan que un DWS «permite a los lexicógrafos codificar los elementos de la macroestructura del diccionario y la microestructura de las entradas desde el inicio, así como proporcionar una variedad de herramientas para facilitar su tarea $\rangle^{2}$ (p. 565).

De acuerdo con lo anterior, puede decirse que un DWS es un software o plataforma diseñada para la compilación, escritura, administración y publicación de diccionarios en distintos formatos. Los DWS también son conocidos con nombres como Dictionary Compilation Software (Joffe \& De Schryver, 2004), Dictionary Building Platform (Mangeot, 2006), Dictionary Content Management System (Alegría, Artola, Astiz \& Ruiz, 2006), Dictionary Editor and Browser o DEB platform (Horák \& Rambousek, 2007), Dictionary Writing and Publishing Systems (Měchura, 2017), entre otros. Estos nombres muestran algunas de las tareas que integran estos sistemas y que varían de acuerdo con los objetivos de sus creadores o con las necesidades de cada diccionario. Actualmente, la mayoría de DWS están diseñados para efectuar todo el proceso de elaboración de diccionarios. Sin embargo, los más completos son los DWS comerciales, que cuentan con todas las herramientas para administrar el proceso completo de elaboración del diccionario, desde su compilación hasta su publicación en formato impreso o electrónico (Atkins \& Rundell, 2008).

En general, un DWS puede incluir una base de datos, un editor o interfaz de edición o varias herramientas administrativas (Kilgarriff, 2006; Atkins \& Rundell, 2008). A continuación, se procede a describir cada uno de estos componentes y sus funcionalidades.

\subsection{La base de datos}

El lenguaje estándar más reconocido para estructurar los datos de los diccionarios es el Extensible Markup

2. «[...] allows lexicographers to encode the elements of the macrostructure of the dictionary and microstructure of the entries for outset, as well as providing a variety of tools to facilitate their task.» (Grundy \& Rawlinson, 2016, p. 565). (Traducción propia). 
DWS y otras herramientas informáticas para la elaboración, administración y publicación de diccionarios Language (XML) (Calzolari et al., 2001, como se citó en Langement, Loopmann \& Viks, 2006). Anteriormente, los primeros Machine-Readable Dictionaries ${ }^{3}$ (MRDs) estructuraban la información de los diccionarios con programas editores de XML. En la actualidad, a través de un marcado XML los datos se organizan de forma jerárquica y se marcan a través de etiquetas; por ejemplo, para ingresar la categoría gramatical de una entrada, se introduce el nombre de la etiqueta y la categoría de la entrada de la siguiente manera: <categoría gramatical $>$ sf $</$ categoría gramatical>. Así, para crear un documento XML bien estructurado - llamado comúnmente «well formed» en inglés — se debe seguir un conjunto de reglas sencillas ${ }^{4}$; sin embargo, un mínimo error sintáctico puede generar fallas (Morgan, 2005).

Aunque los programas de edición XML son eficientes y populares, no necesariamente pueden cumplir con todas las necesidades de un proyecto lexicográfico complejo ni fueron diseñados para la labor lexicográfica (Atkins \& Rundell, 2008; Abel, 2012). De ahí que los editores puedan cometer errores fácilmente y que, en algunos casos, sea necesario que tengan conocimientos básicos de programación, pues los sistemas no tienen interfaces amigables. Los DWS buscan solventar esta problemática, ya que cuentan con una interfaz de edición sobre la que trabajan los editores sin tener contacto directo con la base de datos. En este caso, el sistema se encarga de que la información que se ingresa en la interfaz de edición quede almacenada y organizada automáticamente en la base de datos.

En este punto, las bases de datos son fundamentales, ya que sistematizan tanto la información relacionada con el diccionario (entradas, definiciones, categorías, etc.) como también los datos relacionados con la administración del DWS (usuarios, editores, estado de escritura de las entradas, fechas, etc.). De esta manera, gracias a la base de datos se pueden hacer consultas como las últimas entradas creadas, el equipo de editores y las entradas que han escrito o editado, las entradas que tengan una determinada etiqueta — por ejemplo, las que tengan el campo semántico de «alimentación»—, entre otras.

Por otro lado, existen dos funcionalidades importantes que destacar en los DWS actuales. Primero, que la mayoría se basan en una arquitectura cliente-servidor que facilita el trabajo del lexicógrafo desde cualquier equipo y ubicación vía internet (Abel, 2012). Segundo, permiten exportar el diccionario completo o partes de este en distintos formatos (XML, PDF, RFT, TXT, etc.) (Abel, 2012). Asimismo, la mayoría permiten importar información adicional o de otros diccionarios a la plataforma. Esto puede ser vital para complementar información del diccionario que se esté creando o para editar y publicar un diccionario que se haya llevado a cabo con otros sistemas o formatos menos elaborados, por ejemplo, en Word.

3. Los MRDs son versiones electrónicas de los diccionarios impresos comunes (Fayed, Diaa, Fahmy, Aly, Abdelrazek, Mohsen, y Fayed, Wafaa, 2014).

4. Entre algunas de las reglas que señala Morgan (2005) se encuentran las siguientes: 1) los documentos XML siempre tienen un solo elemento raíz; 2) los elementos siempre deben estar cerrados;3) los atributos de los elementos siempre deben estar citados; y 4) solo puede haber 5 entidades por definición $(<,>, \&$, “, y “). 
DWS y otras herramientas informáticas para la elaboración, administración y publicación de diccionarios

\subsection{La interfaz de edición}

«El editor de entradas del DWS constituye el espacio donde el trabajo esencial de la lexicografía se lleva a cabo» ${ }^{5}$ (Grundy \& Rawlinson, 2016, p. 569), es decir, es la interfaz donde se escriben las entradas del diccionario. Generalmente, la interfaz de edición cuenta con dos ventanas centrales y varios menús con herramientas de edición y búsqueda de las entradas. En las dos ventanas se muestra la misma información en dos vistas diferentes: la vista de diagrama de árbol y la vista WYSIWYG (what you see is what you get) o modo previsualización (Atkins y Rundell, 2008, p. 115). En la figura 1, se muestra la interfaz de edición de TLEX: en la parte izquierda, hay un menú para buscar, crear y eliminar entradas, luego, está la vista de diagrama de árbol y en la parte derecha la vista de previsualización. Asimismo, en la parte de arriba está todo el menú de herramientas de edición, formato, vista, entre otras.

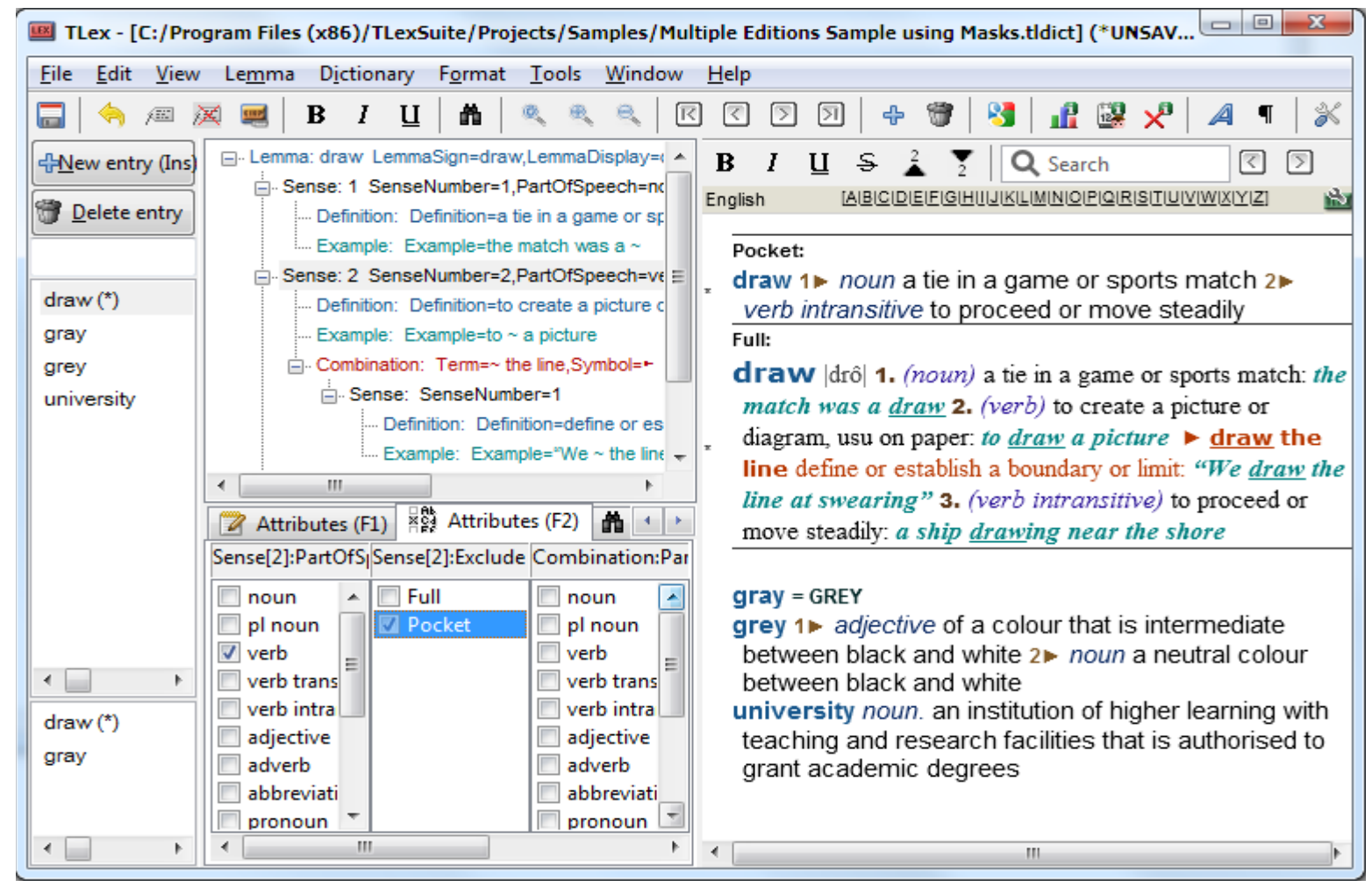

Figura 1. Interfaz de edición de TLex

La ventana de vista de diagrama de árbol (ver Figura 2) también conocido como estructura XML se relaciona con el Document Type Definition (DTD), que «establece las relaciones jerárquicas entre los diversos elementos

5. «The Entry Editor of the DWS constitutes the environment in which the essential work of the lexicography is carried out. » (Grundy \& Rawlinson, 2016, p. 569). (Traducción propia). 
DWS y otras herramientas informáticas para la elaboración, administración y publicación de diccionarios de la entrada» (Grundy \& Rawlinson, 2016, p. 567). En el DTD los lexicógrafos pueden definir cualquier aspecto relacionado con la estructura de la entrada, tales como los elementos que se usarán, el orden permitido en el que ocurran, el contenido que puede aparecer (imágenes, sonidos, caracteres, etc.), los elementos que se usarán reiteradamente y los atributos que tendrá cada elemento o etiqueta (Trap-Jensen, 2013). De esta manera, el editor tiene una estructura ya definida previamente dentro del sistema y los elementos organizados en relación con el DTD.

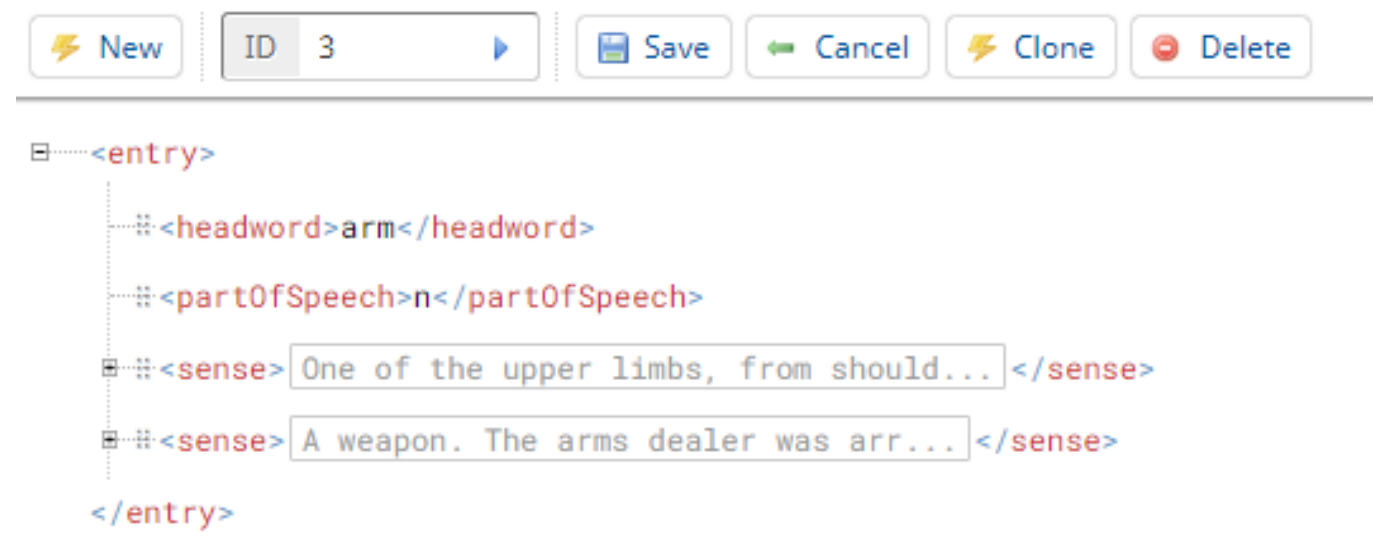

Figura 2. Vista de diagrama de árbol de Lexonomy

Adicionalmente, en la interfaz de edición hay una serie de atributos vinculados a cada etiqueta o elemento de la entrada. El uso de atributos personalizados en XML permite crear grandes listas cerradas para usar en el diccionario, como una de categorías gramaticales (Grundy \& Rawlinson, 2016). De esta manera, el editor puede elegir de un listado desplegable el atributo de la entrada en la que está trabajando, pues tiene la posibilidad de elegir la marca dialectal de una lista ya definida previamente. En la Figura 1, debajo de la vista de árbol se puede ver la ventana para la adición de atributos a la entrada en el programa Tlex.

La ventana WSIWYG, por su parte, permite visualizar el producto final de la entrada en el mismo momento en que se está creando. En este sentido, los DWS cuentan con un espacio para definir cómo se presentarán las entradas del diccionario para su publicación en formato impreso o digital. De esta manera, la información que se visualiza en la Figura 2 se convierte en el panel de previsualización, como se muestra en la Figura 3. Esto permite al editor visualizar el producto de lo que está creando y la verificación constante de posibles errores dentro de la entrada. 


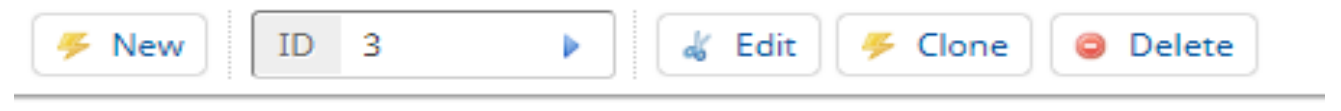

arm $n$

1 One of the upper limbs, from shoulder to wrist.

- You have very long arms .

- My left arm hurts.

2 A weapon.

The arms dealer was arrested.

Figura 3. Ventana de previsualización de Lexonomy

\subsection{Herramientas administrativas}

Los DWS además de facilitar el almacenamiento, escritura y edición del diccionario también cuentan con una serie de herramientas que facilitan la administración de todo el proceso lexicográfico. Algunas de las funcionalidades que proporcionan estas herramientas son:

- Obtener estadísticas sobre todo el proceso lexicográfico, los usuarios, el estado de las entradas, los cambios realizados, las fechas, entre otros. Esto es posible si el DWS cuenta con una función de registro que le permite al lexicógrafo coordinador hacer seguimiento de las entradas y del flujo de trabajo en las diversas fases editoriales (Trap-Jensen, 2013).

- Asignar las entradas en las que debe trabajar cada miembro del equipo y hacer seguimiento de las mismas. Generalmente, los DWS cuentan con herramientas para que el lexicógrafo coordinador realice la revisión y haga comentarios a las entradas directamente en el sistema.

- Asignar determinadas partes de la entrada a especialistas. La mayoría de DWS aseguran que solo una persona trabaje sobre una entrada a la vez y que haya ciertos campos que se puedan bloquear; esto permite que ciertas partes de la entrada sean modificadas solo por expertos (Atkins y Rundell, 2008), por ejemplo, que las etimologías de las entradas solo puedan ser modificadas por la persona asignada y que este campo esté bloqueado para los demás miembros del equipo.

-Definir los permisos de acceso, edición y exportación de datos del diccionario. El sistema debe contar con distintos usuarios y permisos de acceso, de manera que la persona que fue contratada para determinada tarea solo acceda a los espacios e información que necesita para realizar esta tarea, es decir, que el proyecto pueda tener pasantes que solo trabajen en la edición de determinadas entradas o partes de la entrada y que solo tengan acceso 
DWS y otras herramientas informáticas para la elaboración, administración y publicación de diccionarios a los espacios para realizar esta tarea.

- Importar o exportar el diccionario o partes del diccionario en distintos formatos. Esto permitirá que el lexicógrafo editor pueda exportar avances del trabajo realizado o definir los tiempos de adelanto o atraso en la escritura.

- Hacer cambios masivos de los datos a partir de secuencias de comandos, lo que facilita hacer la misma corrección a un conjunto completo de documentos con una sola operación (Abel, 2012).

\section{Corpus Query Systems (CQS)}

Los CQS facilitan la recuperación y análisis de datos de los corpus para la elaboración de diccionarios. Atkins y Rundell (2008) señalan que hay varias decisiones que se deben tomar en el proceso lexicográfico y que muchas de estas se definirán con base en la información que proporcionan los corpus. Por ejemplo, para definir qué entradas se incluirán se puede tener en cuenta la frecuencia de aparición de las palabras dentro del corpus. Es por esto que los CQS deben permitir al lexicógrafo o lingüista expresar las propiedades del fenómeno examinado de la manera más precisa posible para reducir la cantidad de datos que se deben examinar de forma manual (Christ, 1994). En este caso, lo más importante no es si el repertorio léxico contiene toda la información necesaria —que seguramente tendrá si está bien construido-, sino la facilidad y la rapidez con que se pueden recuperar los datos con ayuda del CQS (Atkins \& Rundell, 2008).

Además, los CQS se pueden usar de forma adicional a un DWS o hacer parte de este (Abel, 2012). Mientras que algunos se diseñaron para la consulta de corpus específicos, otros lo fueron para la consulta de varios tipos de repertorios léxicos. Entre algunos de los sistemas no integrados se encuentran los siguientes:

- Sketch Engine ${ }^{6}$, que se refiere a dos aspectos diferentes: el software (CQS) y el servicio web. El primero, toma corpus de cualquier lengua y genera word sketches, además de otros datos. Los word sketches «son resúmenes automáticos de una página con información gramatical y colocacional de una palabra» ${ }^{7}$ (Kilgarriff, Rychly, Smrz, \& Tugwell, 2004. p. 105). El segundo, incluye además del software una gran cantidad de repertorios léxicos precargados y varias herramientas para crear, instalar y administrar conjuntos léxicográficos propios (Kilgarriff, Baisa, Bušta, Jakubíček, Kovář, Michelfeit, \& Suchomel, 2014)

- Emdros ${ }^{8}$ es un motor de base de datos para el análisis y anotación de texto. Se basa en un modelo Extended $\mathrm{MdF}(\mathrm{EMdF})$ que cuenta con cuatro elementos: un monad o número entero; un objeto que es un set de enteros asociados a un objeto tipo; un objeto tipo que son grupos de objetos asociados por categorías, como «palabra»; y un rasgo — cada objeto puede tener un conjunto de rasgos, es decir los rasgos son los atributos del objeto-

6. Programa disponible en: https://www.sketchengine.eu/

7. «Word sketches are one-page automatic, corpus-based summaries of a word s grammatical and collocational behaviour.» (Kilgarriff., Rychly, Smrz, \& Tugwell, 2004. p. 105). (Traducción propia).

8. Programa disponible en: https://emdros.org/ 
DWS y otras herramientas informáticas para la elaboración, administración y publicación de diccionarios (Petersen, 2004).

- $\mathrm{BNCweb}^{9}$ es un programa cliente servidor web que permite buscar y recuperar datos léxicos, gramaticales y textuales del British National Corpus (BNC). Se basa en un procesador de consultas de corpus (CQP) del IMS Open Corpus Workbench para proporcionar diferentes tipos de búsquedas e información sistematizada en una interfaz amigable (Lancaster University, 2008).

—El sistema de búsqueda Browse/Word de los corpus $i$ Web y el Corpus of Contemporary American English $(\mathrm{COCA})^{10}$. Este sistema permite navegar entre las 60.000 palabras principales en el corpus y ver una amplia gama de información sobre cada una de estas palabras (colocaciones, temas, grupos, concordancias, sinónimos, etc.) (COCA, 2020).En las figuras 4 y 5 se muestra el tipo de información que proporciona este sistema de búsqueda sobre el sustantivo inglés thinking.

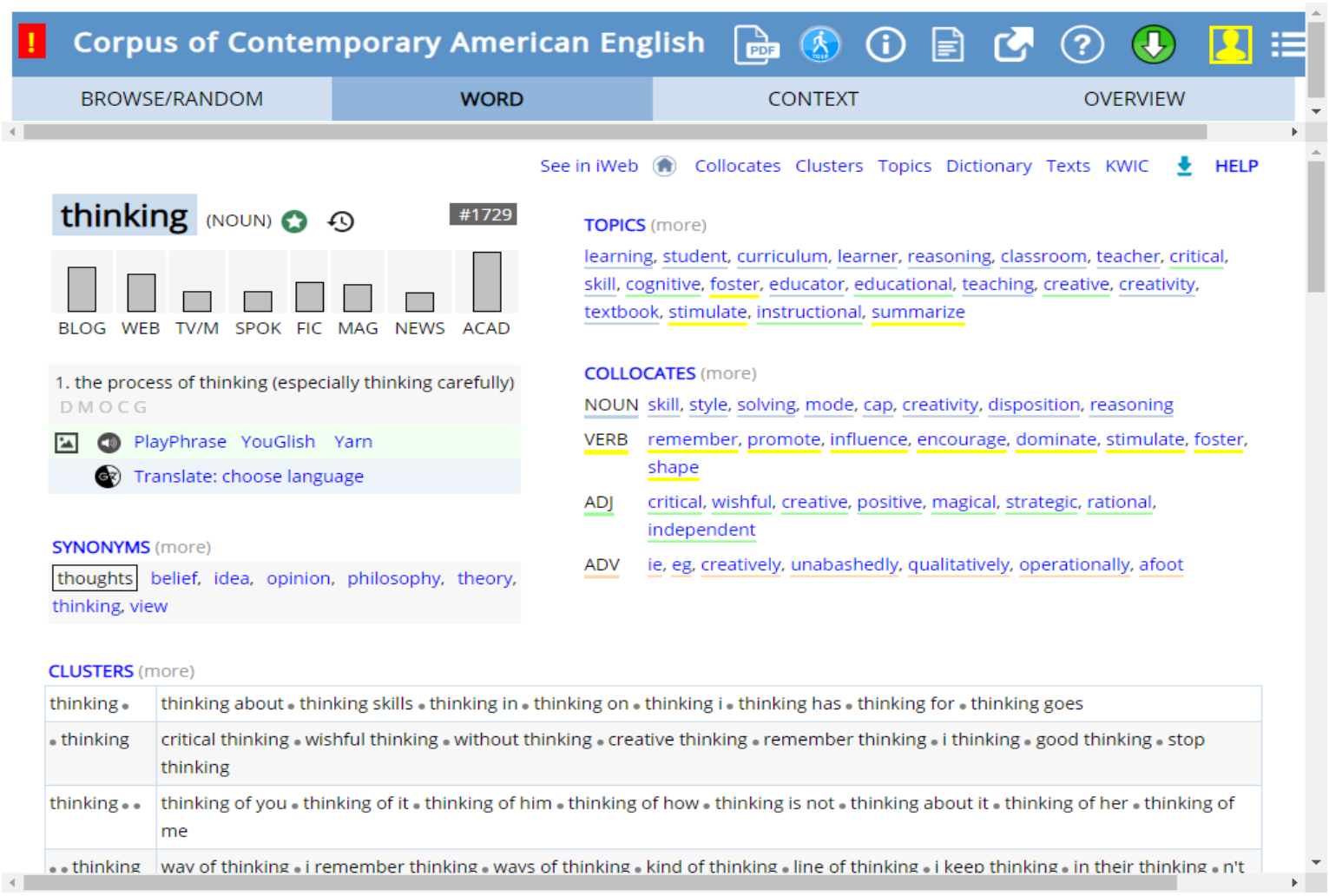

Figura 4. Información del sustantivo inglés thinking en el corpus COCA

9. Programa disponible en: http://corpora.lancs.ac.uk/BNCweb/index.html\#access

10. Programa disponible en: https://www.english-corpora.org/ 
DWS y otras herramientas informáticas para la elaboración, administración y publicación de diccionarios

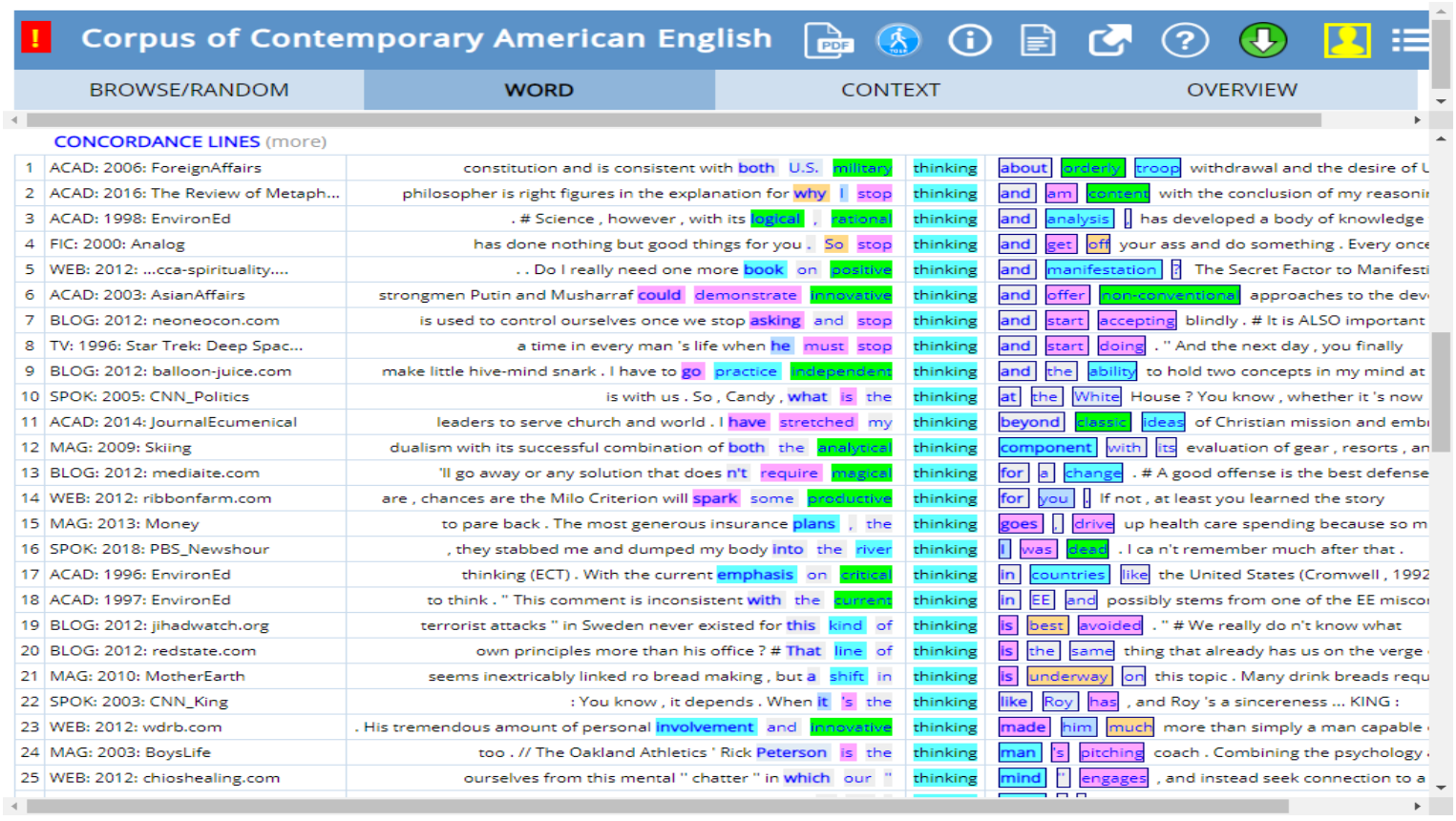

Figura 5. Concordancias del sustantivo inglés thinking en el corpus COCA

En cuanto los DWS que integran un CQS, dentro de su sistema están el TLex y TermStar (ver apartado 3). Asimismo, existen varios sistemas que, aunque no son DWS, permiten la creación de diccionarios y cuentan con un corpus base para llevar a cabo esta tarea, como Sketch Engine.

\section{Tipos de DWS}

Los DWS se pueden clasificar dependiendo de diversos factores, a saber, si son comerciales, propios, o de acceso libre, ya que esto puede influir en la estructura, flexibilidad y funcionalidades que proporcionan. En una cuarta categoría se consideran los aplicativos que fueron diseñados con otros propósitos, pero que de igual modo cuentan con funcionalidades para la creación de diccionarios y listas terminológicas o para realizar cualquier tarea relacionada con el proceso lexicográfico.

\subsection{DWS comerciales}

Los DWS comerciales suelen ser los más completos en cuanto a las herramientas y funcionalidades que proporcionan. Generalmente, cuentan con todos los componentes para la elaboración y publicación de diferentes tipos de diccionarios y, como su nombre indica, son herramientas de pago. Al respecto, Abel (2012) señala el 
DWS y otras herramientas informáticas para la elaboración, administración y publicación de diccionarios hecho inevitable de que las innovaciones positivas sean instauradas por un proveedor y luego sean adoptadas por lo demás, lo que explica que la mayoría de productos comerciales sean de buena calidad.

Entre algunos de los DWS comerciales se encuentran los siguientes:

-TshwaneLex o TLex: ${ }^{11}$ es uno de los programas más utilizados en proyectos lexicográficos en todo el mundo, el cual incluye cuatro herramientas: TLex (DWS), TlTerm (software para la recopilación de listas de terminología), TlCorpus (CQS), y TIReader (herramienta para la visualización del TLex, TlTerm y bases de datos terminológicas). El programa cuenta con varios tipos de licencias: académica, personal, comercial, gubernamental y organizacional (TshwaneDJe, 2003_2020). Asimismo, el sistema permite trabajar con diccionarios monolingües y bilingües en todas las lenguas (ver Figura 6). Se caracteriza por tener una interfaz amigable, así como por brindar las opciones de generar numeración automática de sentidos y de homónimos, hacer seguimiento automático de referencias cruzadas y facilitar la exportación a diferentes formatos, comoWord, RTF, HTML, XML, Adobe InDesign, entre otros (De Schryver, 2011).

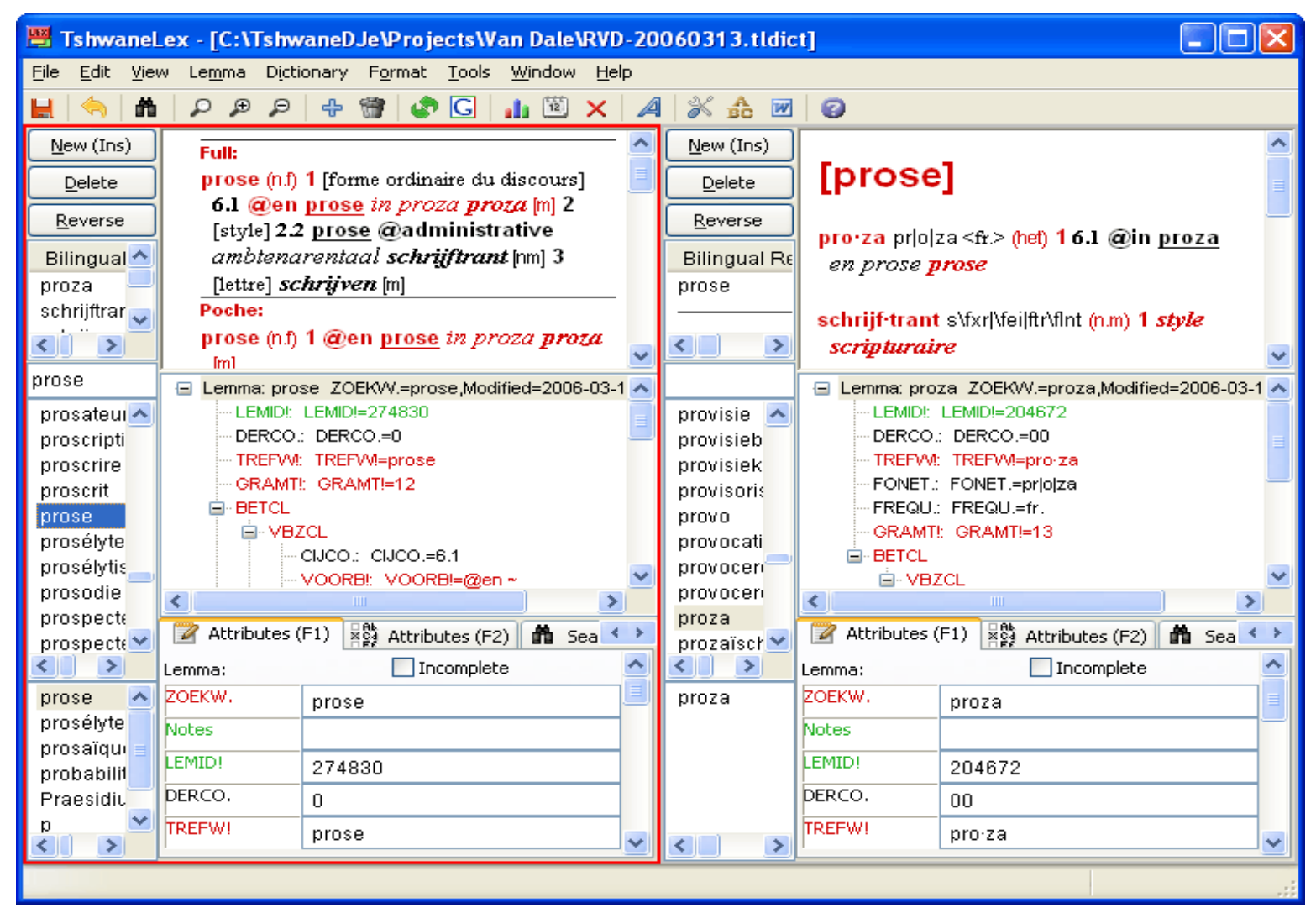

Figura 6. Vista de diccionario bilingüe francés-alemán/alemán-francés Van Dale/Le Robert. Nota: Data Copyright Van Dale: https://shwanedje.com/tshwanelex/overview.html\#bil

11. Programa disponible en: https://tshwanedje.com/tshwanelex/ 
DWS y otras herramientas informáticas para la elaboración, administración y publicación de diccionarios

- ABBYY Lingvo Content: ${ }^{12}$ es un DWS diseñado para compilar diccionarios, glosarios, enciclopedias y otros de materiales de referencia. Es parte de la plataforma Lingvo, que incluye varias herramientas para la conversión de contenido y publicación de diccionarios, lo que permite su publicación en formato electrónico, en papel, o en línea (Rylova, 2010). Entre algunas de las funcionalidades de este sistema están la facilidad para cambiar la estructuración de las entradas, la posibilidad de comparar varios diccionarios, la conformación degrupos de entradas manual o automáticamente y la creación o actualización de diccionarios y su exportación del DWS a una aplicación de usuario final ABBYY Lingvo. además, este programa cuenta con gestión del flujo de trabajo y acceso multiusuario (Kuzmina \& Rylova, 2009).

—Dictionary Production System (DPS): ${ }^{13}$ creado por la compañía francesa IDM (IDM PS), está compuesto por dos componentes principales (IDM France, s.f.): el DPS Website, un sitio web al que se accede desde un navegador normal y en el que se encuentran las herramientas para la gestión de proyectos, la administración y configuración del sistema, desde donde se puede acceder al servidor central donde se almacenan los datos del proyecto. Además, el DPS cuenta con un editor de entradas, que es una aplicación de Windows conectada a la página del programa que permite la descarga de datos del repositorio para la edición de entradas y su carga en el repositorio una vez editadas. Las funcionalidades de este sistema son la gestión y organización de flujo de trabajo; la creación de tareas y lotes y su asignación a miembros del equipo; la creación de reglas secuenciales que el equipo debe cumplir; el acceso a estadísticas de proyecto; la exportación de los resultados obtenidos a diferentes formatos, como XML; el uso de etiquetas, archivos adjuntos y anotaciones, entre otras (IDM France, s.f.).

\subsection{DWS propios}

Antes de que existieran los paquetes completos de DWS comerciales era normal o necesario que los editores de diccionarios desarrollaran sus propias herramientas o adaptaran editores de XML genéricos (Abel, 2012). Por lo cual, muchas casas editoriales o universidades han desarrollado DWS propios que se puedan adaptar a las necesidades de sus proyectos lexicográficos. Entre algunos de estos desarrollos están:

-EELex: ${ }^{14}$ software en línea diseñado por el Institute of the Estonian Language. Este programa cuenta con los tres componentes de los DWS, pues permite realizar correcciones masivas, hacer pruebas de referencias cruzadas y la generación de información morfológica de esta lengua gracias a que cuenta con una base de datos del diccionario básico de estonio (Jürviste, Kallas, Langemets, Tuulik, \& Viks, 2011). Adicionalmente, el sistema permite la publicación de cualquiera de los diccionarios en versión impresa o digital, y cuenta con una interfaz global de consulta que permite la búsqueda de entradas y palabras clave en los diccionarios del sistema (Jürviste

12. Programa disponible en: https://www.abbyy.com/lingvo-mobile-dictionary/

13. Programa disponible en: https://www.idmgroup.com/

14. Programa disponible en: https://eelex.eki.ee/ 
DWS y otras herramientas informáticas para la elaboración, administración y publicación de diccionarios et al., 2011).

-INL-DWS: es un sistema diseñado por el Institute of Dutch Lexicology (INL). Este software se diseñó para el proyecto Algemeen Nederlands Woordenboek (ANW), que es un diccionario académico en línea basado en un corpus del holandés estándar contemporáneo en Holanda y Flandes; sin embargo, se están haciendo los ajustes para que el DWS pueda servir para otros proyectos lexicográficos (Tiberius, Niestadt, \& Schoonheim, 2014). El sistema cuenta con un editor de entradas y con varias herramientas administrativas en una barra que incluye varios enlaces y otras funcionalidades, como la consulta de corpus —el de holandés en Sketch Engine-; manuales, guías de estilo, diccionarios; lista de lemas y estados de construcción, entre otras (Tiberus et al., 2014).

-Dictionary Content Management System (CMS), diseñado en una colaboración entre el Centro de Lingüística Aplicada de Santiago de Cuba y el Grupo IXA de la Universidad del País Vasco para la realización del Diccionario básico escolar $(\mathrm{DBE})^{15}$. El sistema tiene una arquitectura cliente-servidor y usa un editor de XML libre llamado Berkeley $D B X M L$. Asimismo, cuenta con una interfaz gráfica en la que está la búsqueda de lemas, la vista de árbol y la previsualización del diccionario (Alegría et al., 2006).

\subsection{DWS de software libre o abierto}

Los DWS de software libre en la mayoría de los casos son gratuitos y están disponibles en línea para que el usuario se registre o haga su descarga fácilmente. Entre algunos de estos sistemas se encuentran:

-Matapuna: es un DWS completamente en línea diseñado por Dave Moskovitz en colaboración con la Māori Language Commission of New Zealand. Su interfaz se puede usar desde cualquier navegador y cuenta con una funcionalidad de multiusuarios que permite que varias personas ingresen al mismo tiempo y puedan trabajar en el mismo proyecto (Bah, 2010).

-Lexonomy: es una plataforma en línea gratuita diseñada para diccionarios pequeños y medianos. Este DWS busca ser lo más amigable posible y permite desde el inicio definir una estructura XML arbitraria y al final publicar el diccionario en un micrositio de la página (Měchura, 2017). Cuenta con varias herramientas para la configuración y publicación diccionarios, además de facilitar la consulta de corpus para su posterior elaboración.

- Termania: ${ }^{16}$ es un DWS gratuito diseñado por la compañía de software Amebis de Kasmnik (Eslovenia), en cooperación con Trojina (Instituto de Estudios Eslovenos Aplicados) (Krek, 2010). El portal está diseñado para un público general sin conocimientos especializados sobre computación o lexicografía y cuenta con las herramientas básicas para la escritura del diccionario (configuración inicial, búsqueda de entradas, visor de vista de árbol y de previsaulización, etc.). También, permite exportar la información en varios formatos.

- INLEXPO (Interfaz Lexicográfica Polivalente): es un DWS diseñado por la Universidad de Costa Rica

15. Programa disponible en: https://ixa2.si.ehu.es/dbe/index4.html

16. Programa disponible en: https://www.termania.net/uporabnik/prijava 
DWS y otras herramientas informáticas para la elaboración, administración y publicación de diccionarios para la creación, gestión y edición de sus obras lexicográficas. El código del sistema se encuentra libre en la plataforma GitHub ${ }^{17}$., Se caracteriza por contar con un diseño para el trabajo en equipo a través de una conexión LAN o de internet, con opciones de ajuste de usuarios y la conexión a un corpus determinado. Además, permite la publicación de los resultados en varios formatos (Proinnova, s.f.).

-Dictionary Editor and Browser o DEB Platform ${ }^{18}$ : este programa maneja un entorno estricto en la relación cliente-servidor para sistemas generales de escritura de diccionarios y ha permitido implementar varias aplicaciones de Procesamiento de Lenguaje Natural (PLN) (Horák \& Rambousek, 2007). Permite, además, la escritura de diccionarios en cualquier lengua, facilita el trabajo colaborativo y tieneuna interfaz amigable para los usuarios.

-DEBWrite: ${ }^{19}$ es una plataforma en línea basada en el DEB Platform que permite la cooperación entre equipos y facilita la gestión de los derechos de acceso; este programa cuenta con tres niveles de roles de usuarios: gerente, editor y lector; además, posibilita la publicación de diccionarios tanto para lectores humanos como para aplicaciones externas de PLN (Rambousek \& Horák, 2015).

\subsection{Otros DWS y aplicaciones que apoyan los procesos lexicográficos}

Entre los DWS adicionales u otras aplicaciones que apoyan los procesos lexicográficos están las: herramientas diseñadas principalmente para la traducción, que a su vez cuentan con DWS para la construcción de diccionarios o listas terminológicas (1, 2 y 3 en la Tabla 1); herramientas para la consulta y navegación de diccionarios (4 y 5 en la Tabla 1); plataformas para el manejo de datos lingüísticos que cuentan con herramientas para la construcción de diccionarios (6 en la Tabla 1); herramientas para la publicación de diccionarios (7 en la Tabla 1); y DWS para la elaboración de diccionarios de lengua de señas (8 en la Tabla 1).

17. Programa disponible en: https://github.com/LinguisticaUCR/Inlexpo

18. Programa disponible en: https://deb.fi.muni.cz/

19. Programa disponible en: https://deb.fi.muni.cz/proj_debwrite.php 


\begin{tabular}{|c|c|c|c|c|}
\hline Número & Tipo & Nombre & Características & Enlace \\
\hline 1 & $\begin{array}{l}\text { Terminology } \\
\text { management } \\
\text { DWS }\end{array}$ & Multiterm & $\begin{array}{l}\text { Software comercial para la gestión de } \\
\text { terminología. Diseñado para traductores } \\
\text { y terminólogos. Permite personalizar } \\
\text { bases de datos terminológicas con notas, } \\
\text { imágenes, etc., insertararchivos de medios } \\
\text { digitales e hipervínculos; almacenar un } \\
\text { número ilimitado de términos en idiomas; } \\
\text { importar y exportar términos de diferentes } \\
\text { entornos tecnológicos, integrar con SDL } \\
\text { Trados para mejorar la coherencia de la } \\
\text { traducción (Malyar \& Syrvatka, 2015). }\end{array}$ & $\begin{array}{l}\text { https://www.sdl.com/ } \\
\text { software-and-services/ } \\
\text { translation-software/ } \\
\text { terminology-management/ } \\
\text { sdl-multiterm/ }\end{array}$ \\
\hline 2 & $\begin{array}{l}\text { Terminology } \\
\text { management } \\
\text { DWS }\end{array}$ & TermStar & $\begin{array}{l}\text { Software comercial para la gestión } \\
\text { de terminología. Soporta más de } 200 \\
\text { lenguas y se puede vincular, por medio } \\
\text { de complementos, a aplicaciones como } \\
\text { Office. Permite guardar los términos en } \\
\text { dos o más lenguas, elegir la dirección en el } \\
\text { trabajo de traducción, añadir sinónimos, } \\
\text { abreviaturas, notas, imágenes, etc. }\end{array}$ & $\begin{array}{l}\text { http://www.star-transit. } \\
\text { cz/Software/TermStar- } \\
\text { sprava-terminologie/ }\end{array}$ \\
\hline 3 & $\begin{array}{l}\text { Terminology } \\
\text { management } \\
\text { DWS }\end{array}$ & Termkate & $\begin{array}{l}\text { Software gratuito en línea para traducción } \\
\text { que cuenta con funcionalidades para } \\
\text { la elaboración de diccionarios y bases } \\
\text { de datos terminológicas. Permite crear } \\
\text { diccionarios desde cero o importar } \\
\text { diccionarios o listas terminológicas. }\end{array}$ & $\begin{array}{l}\text { https://termkate.elhuyar. } \\
\text { eus/ }\end{array}$ \\
\hline 4 & $\begin{array}{ll}\text { Buscador } & \text { de } \\
\text { diccionarios } & \end{array}$ & GoldenDict & $\begin{array}{l}\text { Software de acceso libre y gratuito, } \\
\text { admite múltiples formatos de diccionarios } \\
\text { electrónicos; permite búsquedas en } \\
\text { Wikipedia, Wiktionary o cualquier } \\
\text { otro sitio basado en MediaWiki; puede } \\
\text { traducir textos largos de muchos idiomas; } \\
\text { permite la consulta de varios diccionarios } \\
\text { a la vez; entre otras (Konstantin, 2008- } \\
\text { 2013). }\end{array}$ & http://goldendict.org/ \\
\hline
\end{tabular}


DWS y otras herramientas informáticas para la elaboración, administración y publicación de diccionarios

\begin{tabular}{|c|c|c|c|c|}
\hline 5 & $\begin{array}{l}\text { Buscador de } \\
\text { diccionarios }\end{array}$ & DEB Dict & $\begin{array}{l}\text { Es una base central de más de } 10 \\
\text { diccionarios, gratuita, tiene una interfaz } \\
\text { de usuarios multilingüe, consulta a varios } \\
\text { diccionarios XML y enciclopedias, } \\
\text { conexión al analizador morfológico } \\
\text { checo, y conexión a un sistema de } \\
\text { información geográfica (Horák \& } \\
\text { Rambousek, 2007). }\end{array}$ & $\begin{array}{l}\text { https://deb.fi.muni.cz/ } \\
\text { proj_debdict.php }\end{array}$ \\
\hline 6 & $\begin{array}{l}\text { Software para el } \\
\text { manejo de datos } \\
\text { lingüísticos/ } \\
\text { DWS }\end{array}$ & $\begin{array}{l}\text { FLEX Fieldworks } \\
\text { Language Explorer }\end{array}$ & $\begin{array}{l}\text { Programa gratuito diseñado por el SIL } \\
\text { International para la recopilación, } \\
\text { análisis, y publicación de datos } \\
\text { en cualquier lengua. En cuanto a } \\
\text { funcionalidades sobre el léxico cuenta } \\
\text { con herramientas para la edición de } \\
\text { diccionarios, edición masiva de entradas, } \\
\text { léxicos inversos, entre otras. }\end{array}$ & $\begin{array}{l}\text { https://software.sil.org/ } \\
\text { fieldworks/ }\end{array}$ \\
\hline 7 & $\begin{array}{l}\text { Plataforma para } \\
\text { la publicación de } \\
\text { diccionarios }\end{array}$ & Lexique Pro & $\begin{array}{l}\text { Software libre desarrollado por el SIL } \\
\text { para la edición y publicación de léxico } \\
\text { interactivo, con hipervínculos entre } \\
\text { entradas, vistas de categoría, inversión de } \\
\text { diccionarios, herramientas de búsqueda } \\
\text { y de exportación. Está diseñado para } \\
\text { mostrar los datos en un formato fácil de } \\
\text { usar para la publicación de diccionarios o } \\
\text { léxicos (Lexique pro, 2012). }\end{array}$ & $\begin{array}{l}\text { http://www.lexiquepro. } \\
\text { com/ }\end{array}$ \\
\hline 8 & $\begin{array}{l}\text { DWS para } \\
\text { diccionarios de } \\
\text { lengua de señas }\end{array}$ & SooSL & $\begin{array}{l}\text { DWS de acceso libre, desarrollado por } \\
\text { el SIL para la elaboración y publicación } \\
\text { de diccionarios de lengua de señas. } \\
\text { Permite trabajar con cualquier lengua de } \\
\text { señas,y el uso de multimedia entre otras } \\
\text { funciones, cuenta con diferentes filtros } \\
\text { para la realización de búsquedas.. }\end{array}$ & https://www.soosl.net/ \\
\hline
\end{tabular}

Tabla 1. DWS adicionales y otras herramientas

\section{Posibilidades e implicaciones del uso de DWS y otras herramientas en los procesos lexicográficos}

El uso de DWS y otras herramientas permite a los lexicógrafos centrarse en su quehacer y los libera de tareas que son fundamentales pero mecánicas (Atkins \& Rundell, 2008). Por tanto, muchas de las labores que los lexicógrafos realizaban de forma manual ahora se pueden realizar automáticamente y con ayuda del computador, lo que también puede prevenir posibles errores humanos. Si, por decir algo, se adicionan nuevas acepciones a una entrada, el DWS se encargará de numerarlas de forma automática. Al respecto, Oppentocht y Schutz (2003) mencionan que: 
Una de las principales limitaciones a las que se enfrentaron los lexicógrafos de los siglos xIX y xx fue la cantidad limitada de espacio en el diccionario de papel. Otra fue el acceso limitado que tenían a los datos del diccionario, ya que solo se podían buscar las palabras clave ordenadas alfabéticamente (p. 216) ${ }^{20}$.

Los DWS han tratado de dar respuestas a la nuevas funcionalidades y necesidades de los diccionarios electrónicos sin omitir las necesarias para la publicación de impresos. En la actualidad, cuando se trata de los primeros,no hay límites en cuanto al espacio que ocupen, por lo que resulta fundamental garantizar que el proceso de consulta sea lo más rápido y fácil posible (Fuertes-Olivera \& Tarp, 2014). Para esto, se plantean varios tipos de consulta que se pueden manejar fácilmente con un buen buscador, pues el sistema contiene toda la información sistematizada en la base de datos. Adicionalmente, los DWS garantizan el almacenamiento de la información a largo plazo y la reutilización de los datos. Frente a esto, Dziemianko (2003) expone que mucha de la información consignada en este tipo de repertorios léxicos puede ser leída no solo por los humanos sino por las máquinas y que estos tienen gran potencial para la investigación lingüística.

Otras de las posibilidades que ofrecen los DWS es la facilidad para el trabajo en equipo, pues, por una parte, la mayoría permiten que los miembros del proyecto trabajen desde cualquier lugar y al mismo tiempo. Por otra parte, las herramientas administrativas facilitan el manejo de las tareas, de los y las entradas; también permiten la revisión y seguimiento del estado del proyecto en todas sus etapas y cuentan con herramientas de comunicación y de revisión internas; además, pueden registrar fechas, participantes y cambios que se realizan a las entradas.

Con respecto al acceso y publicación de los diccionarios, es importante señalar que la mayoría de DWS cuentan con las herramientas para exportarlos en distintos formatos $y$, en algunos casos, cuentan con una interfaz gráfica para su publicación directa. Además, en la actualidad hay una necesidad constante de que este tipo de repertorios léxicos estén disponibles en distintas presentaciones (impresos, en línea o como aplicación móvil). Esto implica que la misma información contenida en la base de datos se pueda adaptar para su publicación en los formatos necesarios. Al respecto, Trap-Jensen (2013) menciona que, si la estructura del diccionario se ha diseñado con el cuidado suficiente, es posible tener en cuenta las diferencias en la fase de publicación.

Ahora bien, además de las nuevas posibilidades que proporcionan los DWS y otras herramientas también hay una serie de implicaciones y nuevas tareas o fases en los procesos lexicográficos. entre las cuales se encuentran las siguientes:

-Decidir qué DWS se usará para la realización del proyecto lexicográfico. Para esto será necesario tener en cuenta varios factores, como el presupuesto, tipo de diccionario, adaptabilidad de la plataforma, necesidades técnicas, etc.

- Adaptar el sistema a todas las necesidades del diccionario y hacer todo el proceso de configuración. En muchos casos, dentro del proyecto lexicográfico, se suele contratar a un encargado técnico de realizar estas tareas.

20. «One of the major limitations that 19th and 20th century lexicographers were confronted with, was the limited amount of space in the paper dictionary. Another was the limited access they had to the data in the dictionary since only alphabetically ordered headwords could be searched for. » (Oppentocht \& Schutz, 2003, p. 216). (Traducción propia). 
DWS y otras herramientas informáticas para la elaboración, administración y publicación de diccionarios Esto dependerá de la amplitud de proyecto y del tipo de accesibilidad de la plataforma elegida.

- Capacitar al equipo en cuanto al uso del DWS y del CQS.

\section{Conclusiones}

En sus inicios, los DWS se diseñaron solamente para apoyar la escritura de las entradas del diccionario, con el tiempo se han convertido en herramientas indispensables que facilitan todo el proceso lexicográfico desde su compilación hasta su publicación en diversos formatos (impreso, en línea o como aplicación móvil). Generalmente están compuestos por tres elementos básicos: la base de datos, la interfaz de edición o de escritura de entradas y un conjunto de herramientas administrativas. Las funcionalidades y herramientas de cada DWS están vinculadas a su tipología y al tipo de diccionarios para el que fueron creados. Los sistemas más completos suelen ser los comerciales, por su competitividad dentro del mercado. Sin embargo, los DWS propios y de software libre también pueden ser muy completos y responder a las necesidades de varios tipos de diccionarios. Además, generalmente son gratuitos.

Junto a los DWS están los CQS, que permiten consultar y analizar grandes cantidades de corpus, pues su principal misión consiste en filtrar la información de estos para la labor lexicográfica. En la actualidad, esta información resulta fundamental para la compilación y escritura de un diccionario, ya que varias decisiones del proceso lexicográfico se toman con base en la información aportada por los repertorios léxicos, como son la elección de las entradas, la identificación de los significados, entre otros.

Hoy en día, el uso de herramientas informáticas no solo ha cambiado los tipos de diccionarios disponibles, sino también los procesos lexicográficos, por lo cual varias de las tareas que se hacían antes de forma manual ahora se hacen con ayuda de los DWS. Esto ha traído varias ventajas en las tareas administrativas, en el almacenamiento y sistematización de la información, en el trabajo en equipo desde cualquier lugar, en las facilidades para la publicación de diccionarios en distintos formatos, en el fácil manejo de la información para la consulta de diccionarios electrónicos y en el aprovechamiento de la información no solo para fines lexicográficos, sino también para tareas de traducción o de investigación lingüística. Asimismo, la automatización del proceso de elaboración de diccionarios ha traído consigo también otras implicaciones para el trabajo lexicográfico, como son la elección del DWS con el que se va a trabajar, la configuración inicial y adaptación del CQS para el diccionario específico y la capacitación de los miembros del equipo en cuanto al uso de estas nuevas herramientas. 
DWS y otras herramientas informáticas para la elaboración, administración y publicación de diccionarios

\section{Referencias bibliográficas}

1. Alegría, I., Arregi, X., Artola, X., Astiz, M., \& Ruiz Miyares, L. (2006). A Dictionary Content Management System. In Atti del XII Congresso Internazionale di Lessicografia, Torino (pp. 6-9). http://ixa.si.ehu.es/sites/ default/files/dokumentuak/3551/dictionary_content.pdf

2. Atkins, B.S. \& Rundell, M. (2008). The Oxford Guide to Practical Lexicography. New York: Oxford University Press.

3. Bah, O. (2010). Review of Matapuna Dictionary Writing System. Language Documentation \& Conservation, 4, 169-176. http://128.171.57.22/bitstream/10125/4477/bah.pdf

4. Christ, O. (1994). A Modular and Flexible Architecture for an Integrated Corpus Query System. Proceeding of COMPLEX'94 $3^{\text {rd }}$ Conference on Computational Lexicography and Text. Budapest, Hungary. (pp. 23-32). arXiv preprint cmp-lg/9408005.

5. COCA (2020). Corpus of Contemporary American English https:/www.english-corpora.org/coca/

6. De Schryver, G. M. (2011). Why Opting for a Dedicated, Professional, Off-The-Shelf Dictionary Writing System Matters? ASIALEX 2011 Proceedings.

7. Durkin, P. (2016). The Oxford Handbook of Lexicography.Oxford: Oxford University Press.

8. Fayed, D., Fahmy, A., Abdelrazek, M., \& Fayed, W. (2014). Towards Structuring an Arabic-English MachineReadable Dictionary Using Parsing Expression Grammars. International Journal of Computational Linguistics Research. http://www.dline.info/jcl/fulltext/v5n1/1.pdf

9. Fuertes-Olivera, P. A. \& Tarp, S. (2014). Theory and Practice of Specialised Online Dictionaries: Lexicography versus Terminography (Vol. 146). Munich: Walter de Gruyter GmbH \& Co KG.

10. Granger, S., \& Paquot, M. (2012). Electronic Lexicography. Oxford University Press.

11. 1IDM France (s.f.). DPS User Manual. http://dps.cw.idm.fr/\#: :text=The\%20Dictionary\%20Production\%20 System\%20(DPS,UK\%2C\%20Europe\%20and\%20the\%20US.

12. Jackson, H. (2013). The Bloomsbury Companion to Lexicography. London: A\&C Black.

13. Joffe, D., \& De Schryver, G. M. (2004). TshwaneLex: A State-Of-The-Art Dictionary Compilation Program. In11th EURALEX International Congress (EURALEX-2004) (pp. 99-104). https://biblio.ugent.be/ publication/299030/file/6778168

14. Jürviste, M., Kallas, J., Langemets, M., Tuulik, M., \& Viks, Ü. (2011). Extending the Functions of the EELex Dictionary Writing System Using the Example of the Basic Estonian Dictionary. eLexicography in the 21st Century: New Applications for New Users, Proceedings of eLex, (pp. 106-112). http://citeseerx.ist.psu.edu/ viewdoc/download?doi=10.1.1.369.3032\&rep=rep1\&type=pdf

16. Horák, A., \& Rambousek, A. (2007). Dictionary Management System for the DEB Development Platform. In Proceedings of the 4th International Workshop on Natural Language Processing and Cognitive Science 
DWS y otras herramientas informáticas para la elaboración, administración y publicación de diccionarios (NLPCS, aka NLUCS) (pp. 129-138). https://www.researchgate.net/profile/Ales_Horak/publication/228908405_ Dictionary_Management_System_for_the_DEB_Development_Platform/links/00b49528f14bb8cca5000000.pdf 17. Krek, S. (2010). Termania-Free On-Line Dictionary Portal. In Proceedings of the $14^{\text {th }}$ Euralex International Congress (pp. 928-930).

18. Kilgarriff, A., Rychly, P., Smrz, P., \& Tugwell, D. (2004). The Sketch Engine. Information Technology. Proceedings of Euralex. (pp. 105-116).

19. Kilgarriff, A., Baisa, V., Bušta, J., Jakubíček, M., Kovář, V., Michelfeit, J., \& Suchomel, V. (2014). The Sketch Engine: Ten Years On. Lexicography, 1(1), 7-36.

20. Konstantin, I. (2008-2013). GoldenDict Dictionary Lookup Program. http://goldendict.org/

21. Lancaster University (2008). BNCweb (CQP-Edition): A Web-Based Interface to the British National Corpus. http://corpora.lancs.ac.uk/BNCweb/

22. Lexique pro (2012). Lexique pro. http://www.lexiquepro.com/

23. Logan, H. M. (1991). Electronic Lexicography. Computers and the Humanities, 25(6), 351-361.

24. Malyar, M. \& Syrvatka, T. (2015). Application of SDL Trados MultiTerm Convert Tools for Termbase Management. Humanity, Computers and Communication (HCC'2015), (pp. 22-24).

25. Měchura, M. (2017). Introducing Lexonomy: An Open-Source Dictionary writing and publishing system. In Electronic Lexicography in the 21st Century: Lexicography from Scratch. Proceedings of the eLex 2017 Conference (pp. 19-21).

26. Meunier, F., De Cock, S., Gilquin, G., \& Paquot, M. (2011). A Taste for Corpora: In Gonour of Sylviane Granger (Vol. 45). Amsterdam: John Benjamins Publishing.

27. Morgan, E. L. (2005). Creating and Managing XML with Open-Source Software. Library Hi Tech, 23(4), 526-540.

28. Petersen, U. (2004). Emdros - A Text Database Engine for Analyzed or Annotated Text. In COLING 2004: Proceedings of the 20th International Conference on Computational Linguistics (pp. 1190-1193).

29. Proinnova Universidad de Costa Rica (s.f.). INLEXPO. http://www.proinnova.ucr.ac.cr/wp-content/ uploads/2016/08/One-Page INLEXPO.pdf

30. Kuzmina, V., \& Rylova, A. (2009). Software Demonstration. The ABBYY Lingvo Electronic Dictionary and the ABBYY Lingvo Content Dictionary Writing System as Lexicographic Tools. eLEX2009, (pp. 419-423).

31. Rambousek, A., \& Horák, A. (2015). DEBWrite: Free Customizable Web-Based Dictionary Writing System. Electronic Lexicography in the 21st century: Linking Lexical Data in the Digital Age (pp. 443-451). Ljubljana/ Brighton: Trojina, Institute for Applied Slovene Studies/Lexical Computing Ltd.,

32. Rylova, A. (2010). Electronic Dictionary and Dictionary Writing System: How this Duo Works for Dictionary User's Needs (ABBY Lingvo and ABBY Lingvo Content case). In Proceedings of the 14th Euralex International Congress (pp. 1105-1111).

33. Tiberius, C., Niestadt, J., Schoonheim, T. (2014): The INL Dictionary Writing System. Slovenščina 2.0, 2(2): 
DWS y otras herramientas informáticas para la elaboración, administración y publicación de diccionarios 72-93. http://www.trojina.org/slovenscina2.0/arhiv/2014/2/Slo2.0_2014_2_06.pdf.

34. TshwaneDJe (2003_2020). TLex Suite: Dictionary Compilation Software. https://tshwanedje.com/tshwanelex/ 35. Van Sterkenburg, P. (2003). A Practical Guide to Lexicography (Vol. 6). Amsterdam: John Benjamins Publishing. 